Original Article

\title{
ASSESSMENT OF MEDICATION ERRORS IN PEDIATRICINPATIENT DEPARTMENT OF A PRIVATE HOSPITAL
}

\author{
P. NIKHITHASRI ${ }^{1}$, M. RAMYA ${ }^{2}$, P. KISHORE ${ }^{*}$ \\ 1Department of Pharmacy Practice, Care College of Pharmacy, Warangal, Telangana, Oglapur (V), Damera (M), Warangal Rural-506006, \\ Telangana ${ }^{2}$ Not affiliated to Any Institution \\ Email: kpcopsaz@gmail.com
}

Received: 20 Aug 2017, Revised and Accepted: 13 Oct 2017

\begin{abstract}
Objective: To assess the overall rate and incidence of medication errors in pediatric inpatients and to determine the importance of pharmacist participation in medication errors.
\end{abstract}

Methods: A prospective observational study has been conducted in a 'private children's hospital' for 6 mo at Warangal, Telangana. Patients who are $\leq 18$ y were considered. Data were collected from patient records, direct communication with the patient and their caregivers.

Results: Among 400 patients with 2,461 medication orders, 1381(56\%) errors were found. Patients were more exposed to AME (33.7) caused by the nursing staff, followed by PME-21.5, CME-0.6, DME- 0.2 in incidence with the $95 \% \mathrm{CI}$.

Conclusion: Pediatric patients are more exposed to administration errors and prescribing errors. Pediatricians and Pharmacists should develop effective programs for safe administration of medications, report medication errors, eliminate barriers in reporting medication errors, encourage a non-punitive reporting culture and create an environment of medication safety for all hospitalized pediatric patients.

Keywords: Medication error, Pediatrics, PME (Prescribing Medication Errors), AME (Administrative Medication Errors), DME (Dispensing Medication Errors), CME (Compliance Medication Errors).

(C) 2017 The Authors. Published by Innovare Academic Sciences Pvt Ltd. This is an open-access article under the CC BY license (http://creativecommons.org/licenses/by/4.0/) DOI: http://dx.doi.org/10.22159/ijcpr.2017v9i6.23432

\section{INTRODUCTION}

'A Medication error is any preventable event that may cause or lead to inappropriate medication use or patient harm while the medication is in the control of the healthcare professional, patient, or consumer. Such events may be related to professional practice, healthcare products, procedures, and systems, including prescribing, order communication product labeling, packaging and nomenclature, compounding. Dispensing, distribution, administration, education, monitoring and use [1].

Pharmaceutical care implicates not only overall medication therapy but also decisions regarding the individualized drug treatment process [2]. The goal of drug therapy process is achieving a defined therapeutic outcome in order to improve the patient quality of life by minimizing the risk. Medication errors compromise the patient's confidence in the health care system [3], it may be committed by both experienced and inexperienced staff, including pharmacists, physicians, nurses, supportive personnel (e. g., pharmacy technicians), administrators, pharmaceutical manufacturers, patients and their caregivers) and others [4]. Medication errors cause more harm in children compared to adult counterparts [5-6] because most pediatric drug doses are calculated based on the age, body weight,body surface area and other clinical conditions. Majority of the drugs used in the children are an unlicensed and off-label and may lead to adverse drug events and sometimes death [7-12]. There are extremely limited number of clinical studies due to different groups in pediatric population which includes from neonates to adolescents and in addition pharmaceutical companies are less keen on conducting the specific clinical trials for pediatric patients as they are more challenging and when compared to adult drug market pediatric drug market is smaller [13]. Medication errors are common, inevitable [14] and can occur throughout the drug treatment process [15] such as prescribing, dispensing, administering, compliance [2].

Prescribing medicationerror (PME) includes improper drug selection (based on known allergies, contraindications/indications), dose/ dosage form/route/rate of administration, or instructions for use of a drug product ordered or authorized by physician (or other legitimate prescriber); off-label drug use, illegible prescriptions or medication orders that lead to errors 4 but the prescribing errors are preventable [16-17] and can be minimized by prescribing skills [17]. Children are particularly prone to dosing errors [18-19] and doses in pediatric patients are calculated by taking age,weight/body surface area into consideration with few formulas 3 such as Clarks formula/fried's formula/Youngs formula/Salsiburg formula/BSA/ Pennas formula/ Dillings formula/Cowlings formula/Bastedo's formula/Anders rule/ $\mathrm{mg} / \mathrm{kg}$ regimen/Ratio-proportion method and Formula method.

Dispensing medication errors (DME) are made by dispensing staff. Administration medication errors (AME) are most common errors [20] and include errors made by nursing staff. In order to avoid these errors, nurses should always ensure the best nursing care $[16,21]$.

Compliance medication errors (CME) occurs due to non adherence of dosage regimen [22].

Clinicians need to make sure that pediatric patients are given proper medicine incoordination with the pharmacist to reduce the polypharmacy and minimize the drug toxicity and provide the patients with the safe and effective drug therapy [23-26].

\section{MATERIALS AND METHODS}

There is a limited literature regarding the impact of a pharmacist's contribution to decreasing medication errors in this high-risk population. The purpose of our study was to record and analyze all interventions during a 6 mo time period in a pediatric hospital to determine the benefit of having a paediatrics-trained clinical pharmacist on the floor.

The main objective of the study is to assess the overall rate of medication errors in pediatric inpatient hospital and pharmacist role in reporting the errors.

A prospective observational study has been conducted in a 'private hospital' for six months at Warangal, Telangana. Hospital has pediatric 
intensive care Unit-1, Pediatric intensive care unit-2, Neonatal intensive care unit, GeneralWard with a well-equipped laboratory. Only inpatients $\leq 18$ y of age both gender are included in the study, Outpatients and Patients above $18 \mathrm{y}$ are excluded from the study. After taking oral informed consent, Data was collected from patient records and direct communication with the patient and their care takers. Parameters such as demographic details of the patients (age, gender, height, weight, place, date of admission, date of surgery, date of discharge), past medical history, Past medical history, Family history, Allergies, Provisional diagnosis, Laboratory investigations, radiological investigations (x-ray, CT-scan etc.), Diagnosis, Treatment, Followup along with Drug-drug interactions are collected in a specific data collection form designed.
In this study, we have categorized medication errors into four different types depending on the persons responsible for the errors. If a physician made an error in diagnosing the disease and writing the prescription, it is considered as prescribing medication error (PME). If a dispensing staff makes an error in dispensing the proper medication it is considered as dispensing medication error (DME).

If a nurse made error in following the prescribers order i.e., administration medication error (AME) and finally if the patient is non-compliant to medication it is considered as compliance medication errors (CME) and these are furthurly dived into subtypes as given in fig. 1. In our study, we used Graph-Pad software to measure confidence interval.

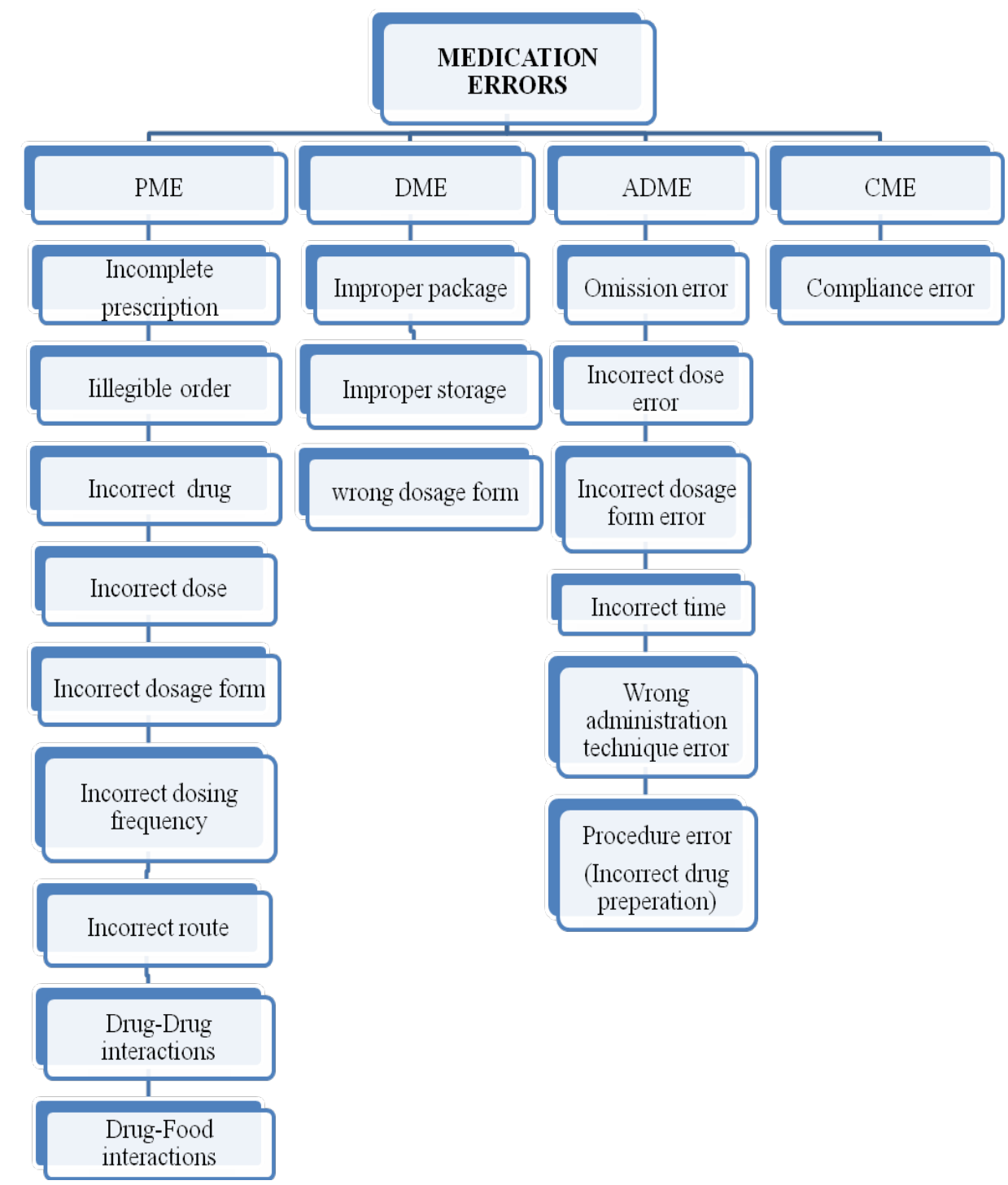

Fig. 1: Types of medication errors [4]

\section{Incorrect drug: Failure to prescribe the correct drug}

Incorrect dose: Prescribing wrong dose instead of correct dose

Incorrect dosage form: Doses administered or dispensed in a different form from that ordered by the prescriber. Dosage form changes may be acceptable to accomdate patient needs. (Ex: Dispensing a liquid formulation without specific prescription to a patient who has difficulty in swallowing tablets might be acceptable to dosage form change).

Incorrect route: Incorrect route of administration of medication.

Incorrect dosing frequency: A physician prescribed BD instead of TID.
Illegible order: Illegible doctors handwriting which may further lead to dispensing errors

Incomplete prescription: Missing the required information in prescription such as ROA, frequency, dose intended prescribed signature.

Drug-drug interactions: occurs when a drug interacts or interferes with another drug.

Drug-food interactions: happen when the food effects the ingredients in the medicine. 
Wrong dosage form: Dispensed wrong dosage form to the patient/ward.

Improper package: Errors in the packaging process for pharmaceutical preparations.

Improper storage: Errors in storage of drugs in a specially designed secure area which may lead to contamination

Omission error: Nurse did not realize drug is due to the patient.

Incorrect dose: Patient is administered the wrong dose instead of the actual dose prescribed by the physician.

Incorrect dosage form: The physical or chemical dosage form integrity has been compromised
Incorrect time: Dose given at wrong time

Wrong administration technique: Doses that are administered using an inappropriate procedure are categorized as wrong administration technique errors.

Procedure error: Drugs requiring reconstitution (adding liquid to the powdered drug) dilution of special preparation prior to dispensing or administration of the drug.

Compliance error: patient's non-compliance to medication.

\section{RESULTS}

In the study period a total of 400 patients were included and prescribed with 2461 medication orders were analysed and 1381 medication errors were found representing $56 \%$.

Table 1: Gender wise distribution of pediatric patients

\begin{tabular}{llll}
\hline Category & Number of male patients (\%) & Number of female patients (\%) & Total (\%) \\
\hline Neonates & $11(2.75 \%)$ & $7(1.75 \%)$ & $18(4.5 \%)$ \\
Infants & $121(30.25 \%)$ & $92(23 \%)$ & $213(53.2 \%)$ \\
Children & $101(25.5 \%)$ & $64(16 \%)$ & $165(41.2 \%)$ \\
Adolescents & $1(0.25 \%)$ & $3(0.75 \%)$ & $4(1 \%)$ \\
Total & $234(58.5 \%)$ & $166(41.5 \%)$ & $400(100 \%)$ \\
\hline
\end{tabular}

In our study male patients (58.5\%) were more compared with female $(41.5 \%)$ and the majority of the patients were infants (53.2\%).

Table 2: Age group distribution of patients with different diseases

\begin{tabular}{|c|c|c|c|c|c|}
\hline Disease & $\begin{array}{l}\text { Neonates } \\
(<27 \text { d })\end{array}$ & $\begin{array}{l}\text { Infants } \\
(<2 y)\end{array}$ & $\begin{array}{l}\text { Children } \\
(2-12 y)\end{array}$ & $\begin{array}{l}\text { Adolscents } \\
(12-18 \mathrm{y})\end{array}$ & Total (\%) \\
\hline Respiratory infections & - & 27 & 44 & - & $71(17.75)$ \\
\hline CNS infections & - & 51 & 31 & 2 & $84(21)$ \\
\hline Blood infections & - & 40 & 18 & - & $58(14.5)$ \\
\hline Viral infections & - & 47 & 25 & 1 & $73(18.25)$ \\
\hline Urinary tract infections & - & - & 3 & - & $3(0.75)$ \\
\hline Coronary heart disease & 4 & 1 & - & - & $5(1.25)$ \\
\hline Anemia & - & 9 & 7 & - & $16(4)$ \\
\hline Surgery & - & 6 & 16 & - & $22(5.5)$ \\
\hline Miscellaneous & 14 & 32 & 21 & 1 & $68(17)$ \\
\hline Total & 18 & 213 & 165 & 4 & $400(100 \%)$ \\
\hline
\end{tabular}

Table 3: Types of error with incidence and \%

\begin{tabular}{|c|c|c|c|c|}
\hline Type of error & No of errors & $\%$ & Incidence & $95 \%$ CI \\
\hline PME & 531 & 38.4 & 21.5 & $19.78-23.49$ \\
\hline DME & 5 & 0.3 & 0.2 & $0.06-0.47$ \\
\hline AME & 830 & 60.1 & 33.7 & $31.47-36.1$ \\
\hline CME & 15 & 1.08 & 0.6 & $0.34-1.0$ \\
\hline Total & 1,381 & $100 \%$ & $56 \%$ & - \\
\hline
\end{tabular}

Above table indicates that patients are more exposed to AME, in which the errors made by the nurses ranked high (i. e wrong time error as the main subtype)

Table 4: Categorization of errors based on age groups

\begin{tabular}{lll}
\hline Age group & No of errors & \% \\
\hline Neonates & 370 & $26.8 \%$ \\
Infants & 613 & $44.3 \%$ \\
Children & 387 & $28.1 \%$ \\
Adolscents & 11 & $0.79 \%$ \\
Total & 1,381 & $99.99 \%$ \\
\hline
\end{tabular}

Among our study population, medication errors were majorly observed in male (i. e $73.4 \%$ ) than females (26.5\%). We observed that patients below $<2$ y were exposed to maximum errors (44.3\%), followed by children (28.1\%), neonates (26.8\%), adolescents $(0.79 \%)$.

Table 5: Gender wise distribution of errors

\begin{tabular}{lll}
\hline Category & Number of errors & Percentage \\
\hline Male & 1014 & $73.4 \%$ \\
Female & 367 & $26.5 \%$ \\
Total & 1,381 & $100 \%$ \\
\hline
\end{tabular}


Table 6: Category wise distribution of different type of errors

\begin{tabular}{|c|c|c|c|c|c|}
\hline Category & PME (\%) & DME (\%) & AME (\%) & CME (\%) & Total (\%) \\
\hline Respiratory infections & $55(10.3)$ & - & $200(24.09)$ & $4(26.6)$ & $259(18.75)$ \\
\hline CNS infections & $77(14.5)$ & $3(60)$ & $187(22.5)$ & - & $267(19.33)$ \\
\hline Blood infections & $49(9.22)$ & $1(20)$ & $125(15.0)$ & - & $175(12.67)$ \\
\hline Viral infections & $55(10.3)$ & - & $82(9.87)$ & $4(26.6)$ & $141(10.20)$ \\
\hline UTI & $3(0.56)$ & - & $1(0.12)$ & - & $4(0.28)$ \\
\hline Coronary heart disease & $7(1.31)$ & - & $3(0.36)$ & - & $10(0.72)$ \\
\hline Anemia & $7(1.31)$ & - & $16(0.722)$ & $2(13.3)$ & $25(1.81)$ \\
\hline Surgery & $15(2.82)$ & - & $32(3.855)$ & - & $47(3.40)$ \\
\hline Miscellaneous* & $49(9.22)$ & $1(20)$ & $157(18.91)$ & $5(33.3)$ & $238(17.23)$ \\
\hline Drug-drug interactions & $214(40.3)$ & - & - & - & $214(15.49)$ \\
\hline Total & $531(38.4)$ & $5(0.3)$ & $830(60.1)$ & $15(1.08)$ & $1,381(100)$ \\
\hline
\end{tabular}

*Miscellaneous include turpentine oil ingestion, pregabalin ingestion, mosquito repellant poisoning, metabolic disorders, orbital cellulitis, hydrocele, acute urticaria, low birth weight, neonatal jaundice, fever and pain in the abdomen.

We found the majority of the study population were more prone to CNS (21.1\%) and respiratory infections (17.1\%).

Among 400 patients and nine different type of category (diseases/ miscellaneous/Drug-Drug interactions/surgery). 1, 381 errors were observed and it demonstrates that AME (60.01\%) is most widely distributed error in all category of diseases followed by PME (38.4\%), CME (1\%) and DME (0.3\%). Medication errors occurred in CNS infections $(19.33 \%)$ were the highest followed by respiratory infections (18.75\%), as mentioned in table 6. Of all errors, Administration errors were the highest and the patients with respiratory infection, CNS infections and blood infections were majorly suffered.

Table 7: Type of error wise distribution in PME

\begin{tabular}{|c|c|c|c|}
\hline Type of error & Number of male patients (\%) & Number of female patients (\%) & Total (\%) \\
\hline Incorrect dose & $175(32.9)$ & $49(9.22)$ & $224(42.18)$ \\
\hline Incorrect dosing frequency & $6(1.12)$ & $3(0.56)$ & $9(1.69)$ \\
\hline Illegible prescriptions & $45(8.47)$ & $15(2.82)$ & $60(11.29)$ \\
\hline Incorrect drug & $4(0.75)$ & $4(0.753)$ & $8(1.50)$ \\
\hline Wrong dosage form & $2(0.37)$ & $1(0.188)$ & $3(0.56)$ \\
\hline Incomplete $\mathrm{Rx}$ & $8(1.5)$ & $5(0.941)$ & $13(3.38)$ \\
\hline Drug-drug interactions & $160(30.13)$ & $54(41.2)$ & $214(40.3)$ \\
\hline Total & $400(75.3 \%)$ & $131(24.6 \%)$ & $531(100 \%)$ \\
\hline
\end{tabular}

Of 1381 medication errors, 531 errors were observed in PME types of errors. Among them incorrect doses are high (42.18\%) followed by drug-drug interactions (40.3\%). It indicates that patients are more exposed to incorrect doses, which shows the cumulative effect on the body. In the therapy process, sub therapeutic doses doesn't produce required therapeutic action which may lead to decreased therapeutic benefit but overdoses cause damage the body hence, appropriate doses have to be administered.

Table 8: Type of error wise distribution in AME

\begin{tabular}{lll}
\hline Type of error & Number of male patients (\%) & Number of female patients (\%) \\
\hline Wrong administration technique & $5(0.8)$ & $3(1.3)$ \\
Procedure error (wrong drug preparation) & $8(1.3)$ & $2(0.8)$ \\
Omission error & $6(0.9)$ & $6(2,6)$ \\
Incorrect time & $582(96.8)$ & $218(95.1)$ \\
Total & $601(72.4)$ & $229(27.5)$ \\
\hline
\end{tabular}

In our study, we observed 830 AME's were observed, of which wrong time constitutes the maximum number of errors (96.3\%), that indicates drug is not given within the predefined time, which lead to missed dose, overdose, underdose.

\section{DME and CME}

We found that $5.1 \%$ improper package errors in DME (Dispensing Medication Error (male $n=4$, female $n=1$ ) and $15 \%$ of $\mathrm{CME}$ (Compliance Medication Error (male $n=9$, female $n=6$ ).

\section{DISCUSSION}

Medication errors were common while prescribing and administering medication to pediatric patients. Most often they do not cause much harm but when they do, they cause inimical effects on healthcare

In the present study, a total of 400 patients data has been collected, observed and analysed from four different wards PICU-I, PICU-II, NICU, GW. A total of 10 physicians and 49 nurses of different grades were involved in prescribing, preparing and administering 2461 medication orders to 400 patients during $24 \mathrm{w}$ of observation.
A study conducted by Mansiparihar et al. reported 35.5\% errors and mentioned that majority of errors occurred in pediatric intensive care units. In our study, we have observed $56 \%$ errors and most of them occurred in pediatric and neonatal intensive care units. Medication errors were common in these units because patients were suffering from serious illness and multiple treatment approaches was opted.

A study conducted by Majed et al., in which 2, 380 medication orders were analyzed1, 333 medication errors were found [56\%(95\%CI)]. Dose errors constituted more than one-third of the errors $(39.4 \%)$, $18 \%$ of errors were committed while prescribing antibiotics. Prescription errors occurred most frequently in infants $(44.5 \%)$ by intravenous routes of administration (50.2\%). Approximately onethird of the errors occurred in PICU (pediatric intensive care unit) i. e $(33.9 \%)$. In the present study, similar results have been found, in which dose errors (16.2\%) are majorly observed in prescribing 
antibiotics (12\%), through IV routes of administration. 35\% of errors occurred in PICU

In our study, of four different categories of errors, we found administration medication errors (AME) are high (830)and the incidence of overall administration error rate is $33.7 \%$ (95\%CI31.47\%-36.1\%), in which wrong time was the most common type of administrative medication error.531 prescribing medication errors (PME) were identified with an error rate of $21.5 \%(95 \% \mathrm{CI}$ $19.78 \%-23.49 \%$ ), in which incorrect dose was the most common type of prescribing error and a study conducted by Maisoonabdulahghaleeb et al., shows that in a total of 444 pediatric patients and 2955 medication orders, there were 391 prescribing errors and the overall prescribing medication error rate is $13.2 \%$ (95\%CI $12.0 \%-14.5 \%$ ). Incomplete prescriptions were the most common type of prescribing error.429 Medication administration errors were identified in 2249 opportunities for error, giving an overall medication administration error rate of $19.1 \%$ of opportunities for error (95\% CI 17.5\%-20.7\%). In which incorrect preparation error $89(20.7 \%)$ is the most common type of error. In both the studies Administration medication errors are high followed by prescribing medication errors.

A study conducted by Kenneth et al. found that 19\% of the medication errors were the dosing errors $(605 / 3216)$. In our study, we have observed $16.2 \%$ of dosing errors. This might be due to an error in dose calculations such as weight-based dosing of drugs in children, high patient volume and limited consultation time.

In our study found, wrong time as the most common type of error which is similar to Kenneth et al. This might be due to the improper nurse-patient ratio (1:9). This might be due to high patient volume and limited

A study conducted by Silva et al. shows that there was at least one dispensing error in each high alert medication with 1, 707 dispensing errors in 2571 prescription orders. In our study, we have observed 5 dispensing errors in 2, 461 medication orders. This might be due to limited pharmacist-nursing staff interaction, short staffed, interruptions during prescribing, dispensing and lookalike/sound-alike medicines.

In our study, we have observed $0.6 \%$ (95\%CI 0.34-1.0\%) compliance errors and there is no sufficient literature to compare the compliance errors. Lack of knowledge regarding the importance of dosage regimen and illiterate patient caretakers are the two reasons for compliance errors.

A study conducted by Folli HL et al. has found 4.5 medication errors per 1, 000 medication orders. Patients aged $2 \mathrm{y}$ and less and patients in intensive care unit received the greatest proportion of errant orders. Neonatal patients received the lowest rate of errant orders. The most common type of error was incorrect dosage, and the most prevalent type of error was over dosage. In the present study, we found that 22.7 medication errors per 1000 medication orders. Patients $<2$ yrs were exposed to a major portion of errors $(44.3 \%)$ and the neonates received the lower number of errors, the most common type of error was wrong time error.

A study conducted by Majed AL et al. found that (64.5\%)most of the errors occurred in males than female (35.5\%), 560 medication errors per 1000 medication orders were identified in their study. Our study also shows the similar findings that majority of the errors occurred in male (73.4\%), 561 medication errors per 1000 medication orders were identified.

A study conducted by DW Bates et al. found that over the study period, 10, 070 medication orders were written, and 530 medications errors were identified (5.3 errors/100 orders), for a mean of 0.3 medication errors per patient-day). In the present study, 2461 medications orders were written and 1381 medication errors were found (56.1errors/100 orders), for a mean of 3.4 medication errors per patient-day)

A study conducted by Mohammed alicheragi et al found that $64.5 \%$ errors were made by nursing staff i.e., administration errors. Which is similar to our study (60.1\%).
In our study, we have found that medication errors occurred in $56.1 \%$ of admissions $(1,381$ errors). Of them, most errors occurred in PICU. Errors committed by nursing staff were maximum constituting $60.1 \%$ and almost half of the medication orders are errors. In the present study, we have found $38.4 \%$ prescribing errors, $0.2 \%$ dispensing errors, $60.1 \%$ administering errors, $0.6 \%$ compliance errors. Highest number of errors were found in administration and the least in dispensing.

Medication errors found to be as follows AME $>\mathrm{PME}>\mathrm{DME}>\mathrm{CME}$. In every pediatric ward.

\section{CONCLUSION}

1. Pediatric patients are more exposed to administration error and their percentages are significantly high.

2. Pediatricians should help hospitals develop effective programs for safe administration of medications, report medication errors, eliminate barriers to reporting medication errors, encourage a nonpunitive reporting culture and create an environment of medication safety for all hospitalized pediatric patients.

3. The development, implementation, and assessment of a computerized physician order entry system, clinical decision-support systems, wardbased clinical pharmacists, and improved communication among physicians, nurses, and pharmacists are recommended.

4. The major role of clinical pharmacists in patient care is collecting the past medical, social history, dosage calculations, monitoring drug therapy, lab parameters, checking for the drug-drug interactions, identifying the adverse drug effects, counselling the patient and caretaker regarding the disease, medication use and lifestyle modifications thereby reducing the medication errors.

6. The pharmacist can play an important role in intervene and act on the prescription/dispensing/administration process and identifying drug-related problems before the injury, can occur. The ultimate goal is to provide children safe and effective drug treatment

7. Large-scale prospective studies are recommended to determine the extent and outcomes of medication errors in pediatric hospitals

\section{LIMITATION}

The present study has some limitations. First, the study was conducted only in one setting that does not represent other health care settings in Warangal. Thus, the findings cannot be generalized. The study period was short $(6 \mathrm{mo})$, and most of the orders were collected during the day shift. It was difficult to collect the orders during the evening and night shifts, during which serious mistakes may occur. There was no follow-up study about the consequences of these errors (i.e., deaths and/or adverse events). The incidence of errors was estimated based on the number of errors and not the number of orders with one or more errors, which might overestimate the incidence of errors in this study, making the comparison with other studies difficult.

\section{CONFLICT OF INTERESTS}

\section{Declared none}

\section{REFERENCES}

1. National co-ordinating council for Medication error reporting and Prevention. Available from: http://www.nccmerp.org/ about-medication-errors. [Last accessed on 10 Jul 2017]

2. American society of hospital pharmacist. AHP: Statement on pharmaceutical care. Am J Hosp Pharm 1993;50:1720-3.

3. American society of hospital pharmacist. ASHP: Guidelines on preventing medication errors in the hospital. Am J Hospharm 1993;50:305-14

4. Maisoon Abdullah Ghaleb, Nick Barber, Bryony Dean Franklin, Ian Chi Kei Wong. The incidence and nature of prescribing and medication administration errors in pediatric inpatients. Arch Dis Child 2010;95:113-8.

5. Marilyn McDougall, Sara Arenas-Lopez. Prescribing unlicensed and off-label medicines in children. BNF Children 2007;18:21-9. 
6. Hugar SM, Suganya M, Vikneshan M, Kiran K. Medication errors and its implications in pediatric dentistry. Indian J Oral Sci 2014;5:27-32.

7. Majed I Al-Jeraisy, Menyfah Q Alanazi, Mostafa A Abolfotouh. Medication prescribing errors in a pediatric inpatient tertiary care setting in Saudi Arabia. BMC Res Notes 2011;4:294.

8. Rashed AN, Wong IC, Cranswick N. Risk factors associated with adverse drug reactions in hospitalized children: international multicentre study. Eur J Clin Pharmacol 2012;68:801-10.

9. Margarita Ruano, Elena Villamanan, Ester Perez. New technologies as a strategy to decrease medication errors: how do they affect adults and children differently? World J Pediatr 2016;12:28-34

10. Michael A, Cimino, RPh MS. Assessing medication prescribing errors in pediatric intensive care units. Pediatr Crit Care Med 2004;5:124-32.

11. Savvato Karavasiliadou, Efstratios Athanasakis. An inside look into the factors contributing to medication errors in the clinical nursing practice". Health Sci J 2014;8:32-44.

12. Al Khaja KA, Al-Ansari TM, Sequeira RP. An evaluation of prescribing errors in primary care in Bahrain. Int J Clin Pharmacol Ther 2005;43:294-301.

13. KE Walsh, R Kaushal, JB Chessar. How to avoid pediatric medication errors: a user's guide to the literature. Arch Dis Child 2005;90:698-702.

14. Kenneth N Barker, Elizabeth A Flynn. Medication errors observed in 36 healthcare facilities. Archintern 2002; 162:1897-903.

15. Lesar TS. Tenfold medication dose prescribing errors. Ann Pharmacother 2002;36:1833-9.

16. Marlene R Miller, Karen A Robinson, Lisa H Lubomski. Medication errors in pediatric care: a systematic review of epidemiology and an evaluation of evidence supporting reduction strategy recommendations. Qual Saf Health Care 2007;16:116-26.

17. Folli HL, Poole RL, Benitz WE, Russo JC. Medication error prevention by clinical pharmacists in two children's hospitals. Pediatrics 1987;79:718-22.

18. Albara Alomari, Val Wilson, Patricia M Davidson, Joanne Lewis. Families, nurses and organizations contributing factors to medication administration error in pediatrics: a literature review. iPDc 2015;5:1-15.

19. Ghaleb MA, Barber N, Franklin BD. A systematic review of medication errors in pediatric patients. Ann Pharmacother 2006;40:1766-76.

20. Jost Kaufmann, Michael Laschat, Frank Wappler. Medication errors in pediatric emergencies. Dtsch ArzteblInt 2012;109:609-16.

21. Michael A Cimino, RPh MS, Mark S. Assessing medication prescribing errors in pediatric intensive care. Pediatr Crit Care Med 2004;5:124-32.

22. Kimland E, Nydert $P$, Odlind V. Paediatric drug use with a focus on off-label prescriptions at the swedish hospitals-a nationwide study. Acta Paediatr 2012;101:772-8.

23. Danielle Taddeo MD, Maud Egedy MD, Jean-Yves Frappier MD. Adherence to treatment in adolescents. Paediatr Child Health 2008;13:1-14.

24. David W Bates, Deborah L Boyle, Martha B Vander Vliet. The relationship between medication errors and adverse drug events. J General Int Med 1995;10:199-205.

25. J McIntyre, S Conroy, An Avery, H Corns, I Choonara. Unlicensed and off-label prescribing of drugs in general practice. Arch Dis Child 2000;83:498-501.

26. Gregory L Kearns, Susan M Abdel-Rahman, Pharm Ds. Developmental pharmacology drug disposition, action, and therapy in infants and children. N Engl J Med 2003;18:349.

27. Imtichoonara, Reagill Anthony Nunn. Drug toxicity and surveillance in children. Br J Clin Pharmacol 1996;42:407-10.

28. Vincent Yeung. Clinical trials in children. Paediatric Drug Handling; 2007. p. 5.

29. Mansi Parihar, Gouriraopassi. Medical errors in pediatric practice. Indian Pediatrics 2008;45:586-9.

30. Mohammad Ali Cheragi, Human Manoocheri, Esmaeil Mohammadnejad. Types and causes of medication errors from nurse's viewpoint. Iran J Nurs Midwifery Res 2013;18:228-31.

31. Maria das Dores Graciano Silva, Mario Borges Rosa, Joaquim Antonio Cesar Mota. Concomitant prescribing and dispensing errors at a Brazilian hospital: a descriptive study. Clinics 2011;66:1691-7. 